\title{
Terahertz Reflectarray for Bidirectional Beam Splitting
}

\author{
Tiaoming Niu, ${ }^{* \dagger}$ Withawat Withayachumnankul, ${ }^{*}$ Derek Abbott* and Christophe Fumeaux* \\ * School of Electrical \& Electronic Engineering, The University of Adelaide, Adelaide, SA 5005 Australia \\ ${ }^{\dagger}$ School of Information Science and Engineering, Lanzhou University, Lanzhou 730000, P. R. China \\ Email: niutm@eleceng.adelaide.edu.au
}

\begin{abstract}
A beam splitter based on the concept of a reflectarray is designed for polarization-dependent bidirectional deflection at 1 THz. A unit cell is composed of two sets of orthogonally oriented dipole resonators arranged in an interlaced triangular lattice. A subarray is constructed from multiple unit cells to create two independent progressive phase distributions to respond to the TE and TM polarized incident waves. Numerical results for the near- and far-field distributions of the reflectarray demonstrate that the proposed structure can separate the polarization components of an incident beam by deflecting them into two different directions, i.e. $-48.6^{\circ}$ and $30^{\circ}$, in plane.
\end{abstract}

\section{INTRODUCTION}

Beam splitters with polarization-dependent properties play an important role in applications requiring high polarization purity or polarization-dependent multiplexing/demultiplexing. Several strategies, such as coupled plasmonic waveguide arrays [1], an asymmetrical directional coupler [2], a magnetically tunable liquid crystal phase grating [3], and metamaterials [4], [5], have been adopted for designing or realizing beam splitters across different spectral ranges. These structures or concepts show the feasibility of beam-splitting in the transmission mode. For the reflection mode, owing to their high efficiency and flat profile, reflectarrays have been applied to achieve polarization-dependent beaming forming functions in the microwave regime [6], [7]. In the terahertz regime, we have demonstrated that a reflectarray with orthogonal interlaced-lattice dipoles can be used to realize a beam splitter with symmetrical polarization-dependent deflections [8]. In this work, we extend the concept to design a beam splitter that separates the orthogonal polarization components of an incident beam by deflecting them asymmetrically towards different angles. This function is achieved by applying two different progressive phase changes for the two interlaced dipole arrays.

\section{Design And Results}

As shown in Fig. 1, a unit cell is composed of two types of orthogonal dipole resonators, each type responding to a particular polarization. The unit cell is made of three layers: dipoles made of gold as the top layer, a polydimethylsiloxane (PDMS) dielectric spacer and a platinum ground plane. The surface impedance model used in [9] is adopted here for these metals and the relative permittivity 2.35 and loss tangent 0.06 of PDMS are determined from independent measurements. For operation at $1 \mathrm{THz}$, the size of the unit cell and the thickness of

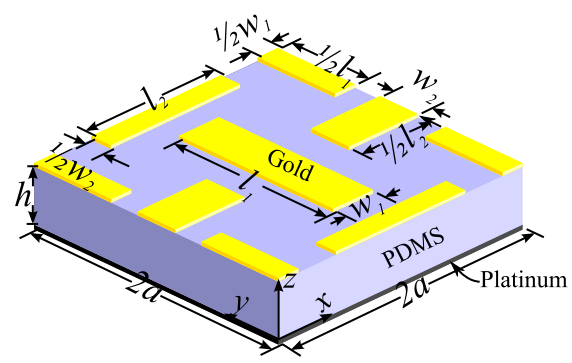

Fig. 1. A structure containing four complete dipoles for the reflectarray.

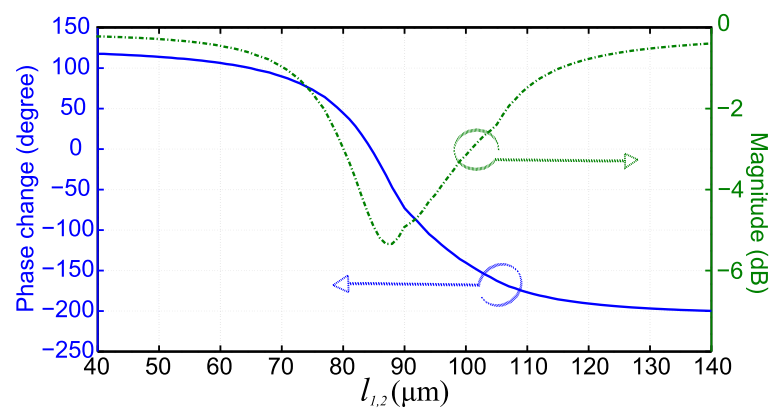

Fig. 2. Reflection response for the structure shown in Fig. 1 at $1 \mathrm{THz}$ as a function of the length $l_{1,2}$, for a dimension $2 a=200 \mu \mathrm{m}$.

the PDMS substrate are selected at fixed values $2 a=200 \mu \mathrm{m}$ and $h=20 \mu \mathrm{m}$, respectively. Different phase responses for a particular polarization can be achieved by varying the length and width of the corresponding dipole. For achieving a smooth phase curve, a piecewise linear function relation between the length and width of the gold dipoles is adopted as in [8]

$$
w_{1,2}= \begin{cases}32 \mu \mathrm{m}+0.2 l_{1,2} & \text { if } 40 \mu \mathrm{m} \leq l_{1,2} \leq 90 \mu \mathrm{m}, \\ 230 \mu \mathrm{m}-2 l_{1,2} & \text { if } 90 \mu \mathrm{m} \leq l_{1,2} \leq 105 \mu \mathrm{m}, \\ 20 \mu \mathrm{m} & \text { if } 105 \mu \mathrm{m} \leq l_{1,2} \leq 140 \mu \mathrm{m} .\end{cases}
$$

With this condition, the numerically determined reflection phase and magnitude response for a uniform array is obtained as shown in Fig. 2. A sufficient phase range of over $300^{\circ}$ and a reasonable reflection efficiency are achieved with the proposed structure.

Based on the phase response data shown in Fig. 2, two different progressive phase changes of $-90^{\circ}$ for the TE wave and $+60^{\circ}$ for the TM wave between adjacent dipoles with the 


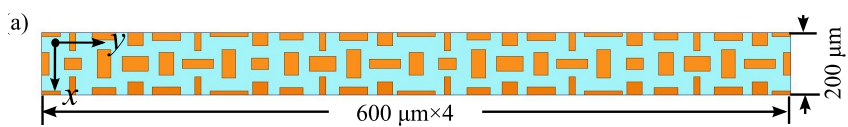

(b)

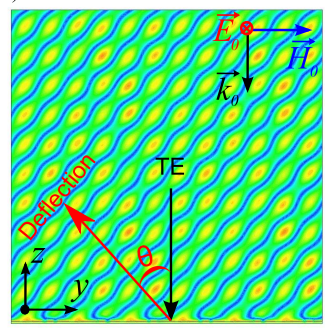

(c)

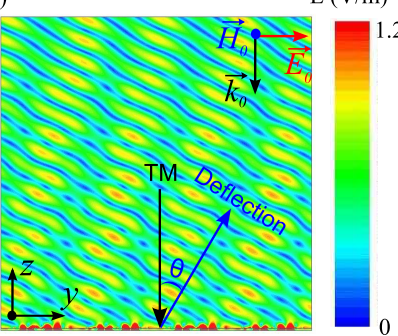

Fig. 3. Reflectarray. (a) Structure of one sub-array made of 48 dipoles (b) and (c) Instantaneous scattered field distributions from the reflectarray at $1 \mathrm{THz}$ for the TE and TM polarizations, respectively. For the normally incident plane wave with the TE and TM polarizations, the reflectarray can deflected them into two different directions with the angles of $48.6^{\circ}$ and $30^{\circ}$, respectively.

same orientation are chosen. This results in a deflection of the incident wave into two different directions. The deflection angle $\theta$ off the specular reflection can be calculated according to the formula $\theta=\arcsin \left(\Delta \phi \lambda_{0} / 2 \pi a\right)$, where $\Delta \phi$ is the progressive phase change and $\lambda=300 \mu \mathrm{m}, a=100 \mu \mathrm{m}$ [9]. With these parameters, the selected progressive phase changes will result in a beam deflection of $-48.6^{\circ}$ and $+30^{\circ}$ for the TE and TM polarizations, respectively. The corresponding sizes of the dipoles for the required phase changes are selected from the simulated phase curve, and the layout of the subarray with realistic material parameters composed of 48 dipoles divided into two groups is configured as shown in Fig. 3(a). The field distributions of the deflected wave at $1 \mathrm{THz}$ for the TE and TM polarizations are demonstrated in Figs. 3(b) and 3(c), respectively. The scattered fields clearly illustrate that the normally incident plane wave is predominantly deflected into the predefined directions according to the incident polarization. In order to demonstrate the ability of a finite reflectarray for beam splitting with bidirectional deflections, we consider a reflectarray that contains $21 \times 252$ subarrays in periodic arrangement. This corresponds to a total sample size of $50.4 \mathrm{~mm} \times 50.4 \mathrm{~mm}$. Based on this configuration, the radiation pattern for the total reflected electrical field can be numerically obtained (neglecting edge effects) using Ansys HFSS as shown in Fig. 4. It is confirmed that the designed reflectarray can separate the polarization components of an incident beam into two different desired directions in a given plane with a polarization purity of $-20 \mathrm{~dB}$ and $-25 \mathrm{~dB}$ for the TE and TM waves, respectively. Due to the asymmetric beam deflections, the detrimental effect from the grating lobes associated with cross-polarization can be reduced.

\section{CONCLUSION}

A terahertz reflectarray with orthogonal strip dipoles in an interlaced triangular-lattice configuration has been proposed for operation as polarization beam splitter. The performance of the proposed structure has been observed through simulations.
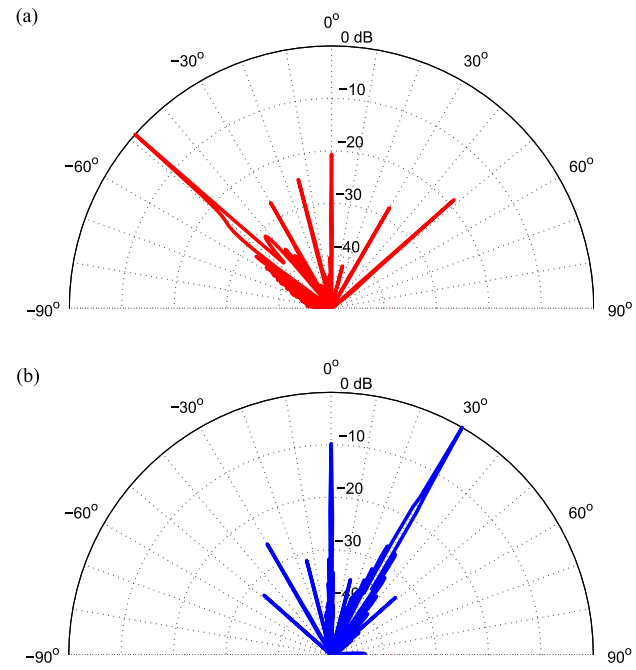

Fig. 4. Simulated radiation patterns of the periodic reflectarray with subarray of Fig. 3(a) and a total size of $50.4 \mathrm{~mm} \times 50.4 \mathrm{~mm}$ for the TE (a) and TM (b) polarized incident plane waves.

The simulation results successfully verify that the designed reflectarray can efficiently and asymmetrically deflect the incident waves with high polarization purity into different directions depending on the incident linear polarization.

\section{ACKNOWLEDGMENTS}

Tiaoming Niu acknowledges support from the Fundamental Research Funds for the Central Universities (No. LZUJBKY2014-43). Derek Abbott and Christophe Fumeaux acknowledge the ARC Future Fellowship funding scheme under FT120100351 and FT100100585, respectively.

\section{REFERENCES}

[1] C. Tai, S. Chang, and T. Chiu, "Design and analysis of an ultra-compact and ultra-wideband polarization beam splitter based on coupled plasmonic waveguide arrays," IEEE Photonics Technol. Lett. 19, 1448-1450 (2007).

[2] X. Guan, H. Wu, Y. Shi, L. Wosinski, and D. Dai, "Ultracompact and broadband polarization beam splitter utilizing the evanescent coupling between a hybrid plasmonic waveguide and a silicon nanowire," Opt. Lett. 38, 3005-3008 (2013).

[3] C. Lin, Y. Li, C. Hsieh, R. Pan, and C. Pan, "Manipulating terahertz wave by a magnetically tunable liquid crystal phase grating," Opt. Express 16, 2995-3001 (2008).

[4] M. Rahm, S. Cummer, D. Schurig, J. B. Pendry, and D. R. Smith, "Optical design of reflectionless complex media by finite embedded coordinate transformations," Phys. Rev. Lett. 100, 063903 (2008).

[5] M. Farmahini-Farahani and H. Mosallaei, "Birefringent reflectarray metasurface for beam engineering in infrared," Opt. Lett. 38, 462-464 (2013).

[6] J. Encinar, L. Datashvili, J. Zornoza, M. Arrebola, M. Sierra-Castañer, J. Besada-Sanmartín, H. Baier, and H. Legay, "Dual-polarization dualcoverage reflectarray for space applications," IEEE Trans. Antennas Propag. 54, 2827-2837 (2006).

[7] J. Perruisseau-Carrier, "Dual-polarized and polarization-flexible reflective cells with dynamic phase control," IEEE Trans. Antennas Propag. 58, 1494-1502 (2010).

[8] T. Niu, W. Withayachumnankul, A. Upadhyay, P. Gutruf, D. Abbott, M. Bhaskaran, S. Sriram, and C. Fumeaux, "Terahertz reflectarray as a polarizing beam splitter," Opt. Express 22, 16148-16160 (2014).

[9] T. Niu, W. Withayachumnankul, B. S.-Y. Ung, H. Menekse, M. Bhaskaran, S. Sriram, and C. Fumeaux, "Experimental demonstration of reflectarray antennas at terahertz frequencies," Opt. Express 21, 28752889 (2013). 